\section{Morphometric assessment of normal, suspect and glaucomatous optic discs with Stratus OCT and HRT II}

ME Iliev, A Meyenberg and JG Garweg
Department of

Ophthalmology, University of Bern, Inselspital, Bern,

Switzerland

Correspondence: ME lliev, Department of

Ophthalmology, University of Bern, Inselspital,

$\mathrm{CH}-3010$ Bern,

Switzerland

Tel: +4131632 2111 ;

Fax. +413138147 79 .

E-mail: milko.iliev@

insel.ch

Received: 21 February 2005 Accepted in revised form:

3 August 2005

Published online:

23 September 2005

The first two authors have contributed equally to this work

\begin{abstract}
Aims To compare morphometric parameters and diagnostic performance of the new Stratus Optical Coherence Tomograph (OCT) Disc mode and the Heidelberg Retina Tomograph (HRT); to evaluate OCT's accuracy in determining optic nerve head (ONH) borders. Methods Controls and patients with ocular hypertension, glaucoma-like discs, and glaucoma were imaged with OCT Disc mode, HRT II, and colour disc photography (DISC-PHOT). In a separate session, automatically depicted $\mathrm{ONH}$ shape and size in OCT were compared with DISC-PHOT, and disc borders adjusted manually where required. In a masked fashion, all print-outs and photographs were studied and discs classified as normal, borderline, and abnormal. The Cohen kappa method was then applied to test for agreement of classification. Bland-Altman analysis was used for comparison of disc measures.

Results In all, 49 eyes were evaluated. Automated disc margin recognition failed in $53 \%$. Misplaced margin points were more frequently found in myopic eyes, but only $31 / 187$ were located in an area of peripapillary atrophy. Agreement of OCT with photography-based diagnosis was excellent in normally looking ONHs, but moderate in discs with large cups, where HRT performed better. OCT values were consistently larger than HRT values for disc and cup area. Compared with HRT, small rim areas and volumes tended to be minimized by OCT, and larger ones to be magnified. Conclusions Stratus OCT Disc protocol performed overall well in differentiating between normal and glaucomatous ONHs. However, failure of disc border recognition
\end{abstract}

was frequently observed, making manual correction necessary. ONH measures cannot be directly compared between HRT and OCT.

Eye (2006) 20, 1288-1299. doi:10.1038/sj.eye.6702101; published online 23 September 2005

Keywords: stratus OCT; optical coherence tomography; scanning laser ophthalmoscopy; glaucoma; optic disc; optic nerve head

Introduction

The clinical relevance of a diagnostic imaging method may be seen in its ability to differentiate between normality and abnormality in borderline cases, where the clinician would appreciate support by objective means. Several imaging systems for the quantitative assessment of morphological changes in glaucoma, that is, of the optic nerve head $(\mathrm{ONH})$ and the retinal nerve fibre layer (RNFL), have been advocated in recent years, the youngest in this application being the optical coherence tomography (OCT). ${ }^{1,2}$ OCT, an optical analogue of B-scan ultrasonography, is based on low-coherence light in the near-infrared spectrum $(840 \mathrm{~nm})$ and creates sagittal optical tissue sections with a resolution of $10-15 \mu \mathrm{m}$ (OCT 2), and $8-10 \mu \mathrm{m}$ (OCT 3) [User manual of Stratus OCT 3000, Carl Zeiss Meditec Inc., Jena, Germany]. ${ }^{3-5}$ OCT is able to measure peripapillary RNFL thickness, and this method has been shown to have good reproducibility, sensitivity, and specificity in differentiating normal from glaucomatous eyes. ${ }^{6-10}$ The most recently launched OCT scanning protocol also performs topographic imaging of the $\mathrm{ONH}$ allowing quantitative (morphometric) analysis. ${ }^{10-12}$ 
The reference system for ONH morphometry in the past 10 years has been scanning laser ophthalmoscopy (SLO) with the Heidelberg Retina Tomograph (HRT), and a huge amount of data about normal and glaucomatous disc topography has thereby been gathered. SLO values have been validated in numerous studies and compared with data from manual disc planimetry and histologybased measurements. ${ }^{13-18}$ Important $\mathrm{ONH}$ parameters such as the disc area have become part of our clinical approach in diagnostic cases. ${ }^{19-23}$ It is therefore indispensable for a new method in this field to be compared with the current standard with respect to both absolute values of the $\mathrm{ONH}$ measures and ability to differentiate between normal and abnormal. Schuman et $a l^{11}$ were the first to address this issue comparing OCT 3 with HRT I. They found good correlation between the two devices, but significant differences in absolute values for given parameters. Intrinsic problems of the automated disc margin recognition in eyes with peripapillary atrophy (PPA) were reported. ${ }^{12}$ Nevertheless, the new mode of OCT seems promising, since it allows operator-free recognition of optic disc borders, and simultaneous evaluation of RNFL and ONH with the same device.

The purpose of this study was to compare the morphometric parameters and diagnostic performance of Stratus OCT 3000 Disc mode and HRT II in normal, suspect and abnormal optic discs. OCT's accuracy in automatically determining $\mathrm{ONH}$ borders and the potential error sources were analysed with the help of time-matched colour disc photographs.

\section{Patients, materials, and methods}

Subjects included in the study underwent imaging of the $\mathrm{ONH}$ with fundus photography, optical coherence tomography, and confocal scanning laser ophthalmoscopy within 1 week, after giving an informed consent. All participants also underwent a full ophthalmologic examination including slit lamp evaluation, gonioscopy, dilated ophthalmoscopy, and repeated white-on-white automated perimetry (Octopus 101, threshold program G2, Haag-Streit International, Koeniz, Switzerland). Conditions that could cause imaging artefacts or diagnostic bias were avoided. Correspondingly, eyes with myopia $<-4.0$ diopters (D), hyperopia $>3.0 \mathrm{D}$, astigmatism $>2.0 \mathrm{D}$, best corrected visual acuity $(B C V A)<0.5(10 / 20)$, moderate or advanced cataract, pseudophakia, pupil diameter $<3 \mathrm{~mm}$, history of diabetes, history of intraocular or neuro-ophthalmologic disorders, or unreliable automated visual fields (reliability factor $>20 \%$ ) were excluded from the study. The purpose was to include eyes with normal, suspicious, and abnormal (glaucomatous) optic discs. The following four clinical categories were allowed:

- normal controls (negative family history of glaucoma, IOP $<20 \mathrm{mmHg}$, normally looking ONH, normal VF),

- ocular hypertension (OHT) (repeated IOP $\geq 22 \mathrm{mmHg}$, open angle, normally looking $\mathrm{ONH}$, normal VF),

- glaucoma suspects because of largely excavated, glaucoma-like discs (but normal IOP and normal VF), and

- glaucoma (confirmed primary open-angle glaucoma with elevated IOP and abnormal VF, that is, reproducible scotomas in the Bjerrum area or nasal depression, or concentric constriction with asymmetry superior/inferior and preserved central VF).

\section{Imaging}

Colour ONH slides, $20^{\circ}$ area (DISC-PHOT), were acquired with a Topcon TRC-50IA fundus camera (distributed by FISBA Optics, St Gallen, Switzerland) after maximal dilatation of pupils with $0.5 \%$ tropicamide and $2.5 \%$ phenylephrine.

HRT (Heidelberg Engineering GmbH, Heidelberg, Germany; software vers. 1.7 of the HRT II) was performed in miosis; the individual corneal curvatures were entered, and headpieces for astigmatic correction were used when appropriate. Images were accepted if standard deviation was $<20 \mu \mathrm{m}$, and confidence interval $<50 \mu \mathrm{m}$. To define the contour line, six or more points were positioned at the inner margin of the scleral ring by an experienced operator. The standard protocol and the extended parameter table were then printed out.

All eyes were scanned in mydriasis with the Stratus OCT 3000 (software version 3.0; Carl Zeiss Meditec Inc., Jena, Germany). Optic disc scans were recorded with optimized z-offset and polarization. The operator was permanently monitoring for steady eye fixation, correct scan position, and good signal-to-noise ratio. Scans were repeated until an image of satisfying quality was obtained. The Fast Optic Disc Scan Protocol (OCT-DISC) was used. The latter protocol performs six cross-sectional radial scans centred on the $\mathrm{ONH}$ and spaced $30^{\circ}$ from one another (Figure 1). Each cross-section is $4 \mathrm{~mm}$ long, and consists of 128 A-scans. The software determines automatically (OCT-DISC aut $_{\text {}}$ ) the disc margin setting a point at the edge of the retinal pigment epithelium (RPE)/choriocapillaris layer on each side of the disc along a cross-section. Disc contour is then extrapolated by the software based on the resulting 12 edge points (Figure $1 \mathrm{Ab}-\mathrm{Cb}$ ). In each cross-section, the two edge points are connected with a blue line, then a red line is placed $150 \mu \mathrm{m}$ above (standard cup-offset), and the cup contour positioned 

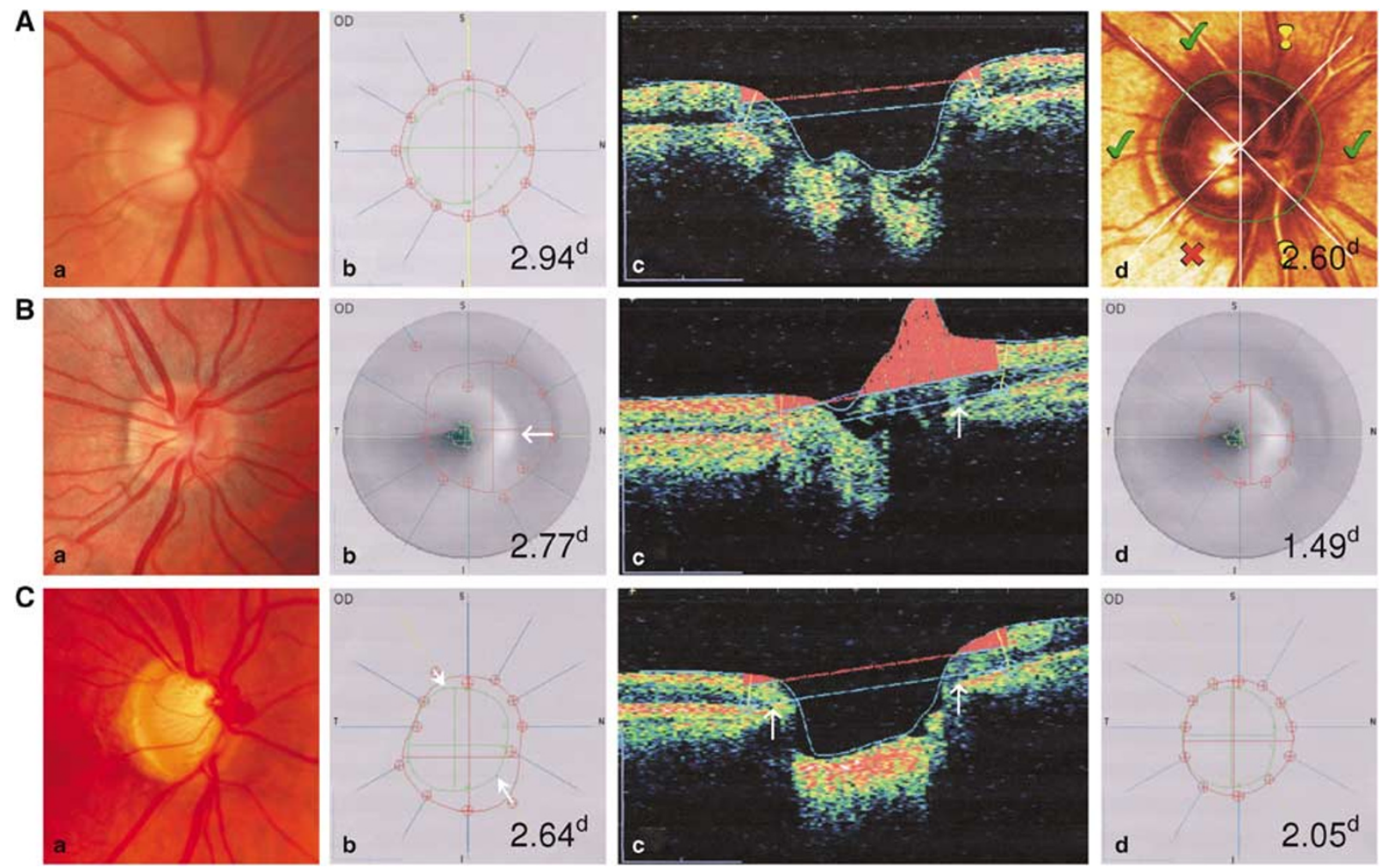

Figure 1 Automatic and manual determination of optic disc margin in OCT, Fast Optic Disc Scan Protocol. Illustration of three optic discs (A, B and C) as depicted in the colour ONH photographs (DISC-PHOT: Aa-Ca), in the OCT mode with automatic disc margin recognition (OCT-DISC aut: disc and cup contour drawing, $\mathrm{Ab}-\mathrm{Cb}$; cross-sectional scan along the yellow meridian, $\mathrm{Ac}-\mathrm{Cc}$ ), and after

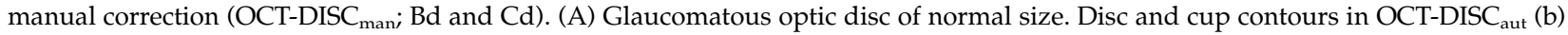
are highly congruent with those appreciated in the photograph. Note that the peripapillary atrophy (PPA) temporal and inferotemporal is not associated with wrong disc margin determination. Since no manual correction was necessary, the corresponding Heidelberg Retina Tomograph image is shown in d. (B) Normal optic disc of relatively small size. There is a misinterpretation of disc

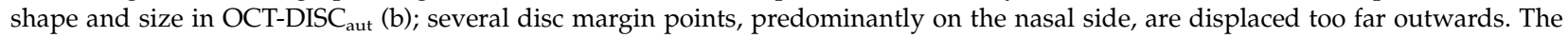
white arrow denotes the manual correction that was carried out along the corresponding meridian. In (c), the original disc margin point position (encircled blue cross) and the manually chosen one (white arrow) are shown. After manual adjustment (d), disc shape and size approximate very well the one in the photograph (a). Regard the considerable change in disc area, and also the absence of PPA. (C) Glaucomatous optic disc of normal size (but smaller than the one in Aa). There is an apparent misinterpretation of disc shape

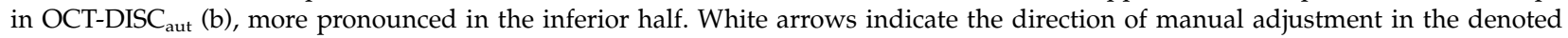
meridian. (c) Cross section along the yellow meridian; right-hand side corresponds to infero-nasal, left-hand side to supero-temporal in (b). The true edges of the retinal pigment epithelium (RPE/choriocapillaris complex), marked with arrows, have not been recognized by the automatic mode. After manual correction (d), a marked improvement, and a good disc shape congruence with the photograph can be appreciated. In this case, PPA may have played a role for disc margin misalignment in the temporal but not in the infero-nasal sector. ${ }^{\mathrm{d}}$ Disc area $\left(\mathrm{mm}^{2}\right)$.

at the crossing of this offset-line with the rim slopes (Figure $1 \mathrm{Ac}-\mathrm{Cc}$ ).

\section{Manual OCT session}

Since automatic determination of $\mathrm{ONH}$ borders resulted in some cases in discs of irregular and unnatural shape and size, in a separate session, all discs and scans per disc were studied and manually redrawn (OCT-DISC man $_{\text {) }}$ when appropriate by one of the authors (AM; Figure 1Bd, Cd). Cup-offset was left unchanged. The colour photographs were used as reference for this session. Disc shape as originally depicted by OCT-DISC aut $_{\text {was }}$ classified as either congruent or mildly, moderately or severely incongruent with the one in the photographs. To evaluate for potential error sources in the automated mode, disc area change after manual fitting, number of corrected margin points per eye, and number of corrected points within the PPA area were recorded, and eyes were divided into two groups: with none or mild shape incongruence, and disc area change after manual fitting $<20 \%$ (good-congruence group); and with moderate or severe shape incongruence and/or disc area change $\geq 20 \%$ (bad-congruence group). 


\section{Image-based classification of $\mathrm{ONH}$}

Apart from comparing the numeric disc parameters, we looked at the overall performance of the two imaging systems in classifying an optic disc as normal or abnormal taking the disc photograph evaluation as a reference. At 4 weeks after the last patient had completed the study, all colour slides were evaluated in a masked, random fashion by one of the authors (MI). Discs were classified as normal, borderline, or abnormal (glaucomatous). After another 4 weeks, print-outs of HRT, OCT-DISC aut $_{\text {, }}$ and OCT-DISC man $_{\text {were classified as }}$ normal, borderline or abnormal independently by two of the authors (MI and AM) according to the discriminating citeria given in Table 1. In cases of disagreement (11 eyes), the masked print-outs were studied by all three authors together, and a final decision was taken.

\section{Comparison and statistical analysis}

Disc shape (reference: DISC-PHOT) and disc area

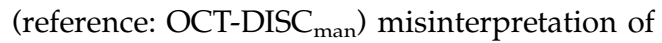
OCT-DISC $_{\text {aut }}$ as well as potential error sources in OCT automated mode were analysed by using the $\chi^{2}$ test, the Fisher exact test, and the unpaired $t$-test. Bland-Altman analysis was performed to investigate agreement of ONH measures between OCT-DISC and HRT. ${ }^{24}$ Association between the two related variables was expressed by the Pearson correlation coefficient $r$, and $P$-values for $r$ were calculated using the $t$-approximation. Differences in $\mathrm{ONH}$ measures between the four diagnostic entities (normal controls, ocular hypertensives, glaucoma-like discs, and glaucoma) were tested using one-way analysis of variance (ANOVA) with the Scheffé multiple contrast method. The Cohen kappa method was used for assessing agreement in $\mathrm{ONH}$ classification (normal, borderline, or abnormal) between imaging systems. ${ }^{25}$ Weighted kappa values were interpreted according to Landis and Koch. ${ }^{26}$ Statistical significance was considered if $P<0.05$.

\section{Results}

A total of 54 eyes of 31 individuals were included in the study. Eight participants were healthy members of the department; for their convenience, both eyes were examined but only one eye was dilated and imaged. All other individuals were regular patients recruited during their outpatient visits. Five eyes had to be excluded prior to analysis because of unreliable visual fields (2), unacceptable quality of disc photographs (1), and HRT images (2). Thus, 49 eyes were available for the final analysis. The demographic and clinical characteristics are summarized in Table 2.

\section{OCT disc margin recognition}

When comparing automatically defined OCT disc shape with the one in the colour disc photographs (Table 3), 10 of $49 \mathrm{ONHs}$ were considered congruent (no manual correction required), 13 showed only mild shape discrepancy $(10+13=23$ eyes in the good-congruence group); 18 eyes exhibited moderate to severe shape incongruence - that is, horizontally instead of vertically oval; with an unnaturally projecting circumference, etc (Figure 1) - and another eight discs presented with a

Table 1 Criteria for classification of ONH with HRT and OCT

\begin{tabular}{|c|c|c|}
\hline Discriminating criteria & Abnormal & Borderline \\
\hline \multicolumn{3}{|l|}{ HRT } \\
\hline $\begin{array}{l}\text { Moorfields classification (at least superior or } \\
\text { inferior quadrant) }\end{array}$ & Outside normal limits & Borderline \\
\hline Depression of green contour line below the zero level & Clear-cut $^{\mathrm{a}}$ & Mild in one or more quadrants \\
\hline $\begin{array}{l}\text { Rim area }\left(\mathrm{mm}^{2}\right) \text { for discs of normal size (disc area } \\
\left.1.6-2.8 \mathrm{~mm}^{2}\right)\end{array}$ & $<1.00$ & $1.00-1.10$ \\
\hline Rim area $\left(\mathrm{mm}^{2}\right)$ for discs of large size (disc area $>2.8 \mathrm{~mm}^{2}$ ) & $<1.10$ & $1.10-1.20$ \\
\hline FSM discriminant function (Mikelberg) & $<0.0$ & $<0.0$ (like abnormal) \\
\hline \multicolumn{3}{|l|}{ OCT-DISC } \\
\hline Rim area $\left(\mathrm{mm}^{2}\right)$ for discs of normal size (disc area $\left.1.6-2.8 \mathrm{~mm}^{2}\right)$ & $<1.00$ & $1.00-1.10$ \\
\hline Rim area $\left(\mathrm{mm}^{2}\right)$ for discs of large size $\left(\right.$ disc area $\left.>2.8 \mathrm{~mm}^{2}\right)$ & $<1.10$ & $1.10-1.20$ \\
\hline Vertical cup-to-disc ratio for normal size disc & $>0.7$ & $0.61-0.7$ \\
\hline Cup shape (as depicted in the OCT print-out) & Clearly vertically elongated & Slightly vertically elongated \\
\hline Rim width/configuration & $\begin{array}{l}\text { Thinnest superior or } \\
\text { inferior/notch }\end{array}$ & Not obeying the ISNT rule ${ }^{\mathrm{b}}$ \\
\hline
\end{tabular}

$\mathrm{ONH}=$ optic nerve head, HRT = Heidelberg Retina Tomograph II, OCT-DISC $=$ ONH imaging and analysis by optical coherence tomography (disc mode of Stratus OCT 3000).

'Both 'humps' flattened below the zero line or a strong asymmetry, the flattened 'hump' correspondig to the thinner rim sector.

${ }^{\mathrm{b}}$ ISNT rule $=$ rim width inferior $>$ superior $>$ nasal $>$ temporal. 
Table 2 Demographic and clinical characteristics

\begin{tabular}{|c|c|c|c|c|}
\hline & Normal & Ocular hypertensives & Glaucoma-like discs & Glaucoma \\
\hline \multicolumn{5}{|l|}{ Demographic characteristics } \\
\hline Eyes/subjects $(n)$ & $14 / 11$ & $9 / 5$ & $9 / 5$ & $17 / 10$ \\
\hline Female/male $(n)$ & $7 / 4$ & $1 / 4$ & $3 / 2$ & $7 / 3$ \\
\hline Age (years); mean (SD) & $43.6(14.5)$ & $56.8(13.6)$ & $52.8(11.1)$ & $68.4(8.5)$ \\
\hline \multicolumn{5}{|l|}{ Clinical characteristics; mean (SD) } \\
\hline BCVA (decimal); mean (range) & $1.29(1.0-1.6)$ & $1.10(1.0-1.6)$ & $1.07(1.0-1.6)$ & $0.92(0.5-1.25)$ \\
\hline Refraction (SE in D) & $-0.98(1.12)$ & $0.29(0.77)$ & $0.07(0.48)$ & $0.35(1.80)$ \\
\hline Mean defect Octopus VF (dB) & $0.69(1.25)$ & $-0.73(0.47)$ & $0.50(1.55)$ & $3.67(3.22)$ \\
\hline Loss variance Octopus VF $\left(\mathrm{dB}^{2}\right)$ & $3.23(1.23)$ & $3.00(1.08)$ & $3.40(1.02)$ & $23.64(16.79)$ \\
\hline Peak IOP $(\mathrm{mmHg})$ & $14.4(3.0)$ & $27.7(3.7)$ & $14.8(1.9)$ & $26.1(7.8)$ \\
\hline Central corneal thickness $(\mu \mathrm{m})$ & $545(38)$ & $587(28)$ & $585(46)$ & $561(29)$ \\
\hline
\end{tabular}

$\mathrm{BCVA}=$ best corrected visual acuity calculated by $\log \mathrm{MAR}(\log$ of the minimum angle of resolution) conversion, $\mathrm{SE}=\mathrm{spherical}$ equivalent, $\mathrm{D}=\mathrm{diopters}$, $\mathrm{IOP}=$ intraocular pressure, Octopus $\mathrm{VF}=$ visual field in automated perimetry (Octopus 101).

Table 3 Factors associated with failure of automatic disc margin recognition in OCT

\begin{tabular}{|c|c|c|c|}
\hline & $\begin{array}{l}\text { OCT-DISC }_{\text {aut }} \text { with good } \\
\text { congruence }^{\mathrm{a}}(\mathrm{n}=23)\end{array}$ & $\begin{array}{c}\text { OCT-DISC }_{\text {aut }} \text { with } \\
\text { bad congruence }(\mathrm{n}=26)\end{array}$ & P-value \\
\hline \multicolumn{4}{|l|}{ Disc area $\left(\mathrm{mm}^{2}\right)$; Mean $(S D)$} \\
\hline OCT-DISC $_{\text {aut }}$ & $2.35(0.53)$ & $2.74(0.51)$ & $0.01^{\mathrm{b}}$ \\
\hline OCT-DISC $_{\text {man }}$ & $2.21(0.43)$ & $2.12(0.32)$ & $0.37^{\mathrm{b}}$ \\
\hline HRT & $2.00(0.40)$ & $2.00(0.38)$ & $0.99^{\mathrm{b}}$ \\
\hline \multicolumn{4}{|l|}{ Peripapillary atrophy (PPA) } \\
\hline Eyes with PPA (n) & 7 & 13 & $0.27^{c}$ \\
\hline $\begin{array}{l}\text { Corrected points within the PPA area/total } \\
\text { number of corrected points }\end{array}$ & $3 / 55(5 \%)$ & $28 / 132(21 \%)$ & $0.01^{\mathrm{d}}$ \\
\hline Refraction $^{\mathrm{e}}$ (D); Mean (SD) & $+0.30(1.23)$ & $-0.44(1.43)$ & $0.06^{\mathrm{b}}$ \\
\hline \multicolumn{4}{|l|}{ ONH classification ${ }^{\mathrm{f}} ;(n)$} \\
\hline Normal & 13 & 11 & $0.48^{\mathrm{c}}$ \\
\hline Borderline and abnormal & 10 & 15 & \\
\hline \multicolumn{4}{|l|}{ Clinical diagnosis; $(n)$} \\
\hline Non-glaucoma ${ }^{\mathrm{g}}$ & 16 & 16 & $0.77^{\mathrm{c}}$ \\
\hline Glaucoma & 7 & 10 & \\
\hline
\end{tabular}

For abbreviations, see Figure 1 and Table 1.

${ }^{\text {a Disc shape was compared between OCT-DISC }}$ aut and the colour optic disc slides. After manual correction of disc borders, the change in disc area was noted in $\%$. To evaluate for potential error sources of failed automatic disc margin detection, two groups were compared: no or mild disc shape error and disc area change $<20 \%$ (good congruence), and moderate or severe disc shape error and/or disc area change $\geq 20 \%$ (bad congruence).

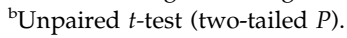

${ }^{\mathrm{c}} \chi^{2}$ test.

${ }^{\mathrm{d}}$ Fisher exact test (two-tailed $P$ ).

e Refraction $=$ spherical equivalent in diopters (D).

${ }^{\mathrm{f}} \mathrm{ONH}$ classification based on colour optic disc slides.

gNon-glaucoma includes normal, ocular hypertensives and glaucoma-like discs.

satisfactorily depicted shape, but an apparently enlarged size $(18+8=26$ eyes in the bad-congruence group). A total of 187 points (ie, $32 \%$ of a maximum of $49 \times 12=588$ points) were manually corrected, mean 2.4 points/eye in the good-congruence group, and 5.1 points/eye in the bad-congruence group $(P<0.001)$. The vast majority of incorrectly determined disc margin points were positioned outside the evident edge of the RPE/ choriocapillaris complex, leading to an 'enlargement' of the disc. After manual correction, the mean decrease of OCT disc area was $5.8 \%$ in the good-congruence group, and $31.2 \%$ in the bad-congruence group $(P<0.0001)$, and disc size did not differ between the groups any more $(P=0.37)$.

The following parameters were assessed for association with disc margin misplacement: 'true' disc area (ie, in 
OCT-DISC $_{\text {man }}$ and in HRT), ONH classification (ie, normal or borderline and abnormal), clinical diagnosis, refraction, and peripapillary atrophy. Only the latter two tended to be correlated with automated disc margin recognition failure. Mean refraction was more myopic in the bad-congruence group $(P=0.06)$. This trend was supported by the finding of a significant negative correlation between refraction and disc area change after manual correction (Pearson $r=-0.31 ; P=0.03$ ). PPA, on the one hand, was significantly more frequently associated with misplaced disc margin points in the badcongruence group than in the good-congruence group (28/132 vs $3 / 55, P=0.01)$; on the other hand, there were only 13 out of 26 eyes in the former group that had any PPA at all, and only $21 \%$ of the misplaced points were located in the PPA area (Table 3).

\section{Agreement of ONH measures between OCT and HRT}

When all 49 eyes were considered, following correlation coefficients $(r)$ were found: OCT-DISC ${ }_{\text {aut }}$ vs HRT: 0.50 for disc area, 0.47 for rim area, and 0.39 for rim volume; OCT-DISC $_{\text {man }}$ vs HRT: 0.67 for disc area, 0.70 for rim area and 0.43 for rim volume ( $P<0.02$ for all comparisons).

Bland-Altman analysis was performed to investigate agreement between OCT and HRT measures (Figure 2). Considerable systematic error and variation were observed. In the scatter plot, OCT-DISC aut $_{\text {disc areas }}$ (Figure 2Aa, black circles) were clearly larger than those of HRT, almost all data points lying above the equality line. Bias and variation were reduced for OCT-DISC Oman $_{\text {}}$ (white circles), but they still remained within a considerable range.

Considering rim area and, particularly, rim volume, a proportional error between both devices was reflected in all plots (Figure $2 \mathrm{Ab}-\mathrm{Cb}, \mathrm{Ac}-\mathrm{Cc}$ ), that is, divergence tended to change according to a parameter's absolute value. Discs with small rim areas $\left(<1.10 \mathrm{~mm}^{2}\right)$ and volumes $\left(<0.24 \mathrm{~mm}^{3}\right)$ - that is, suggestive for glaucoma - tended to show lower values in OCT than in HRT, whereas in ONHs with large rim areas and volumes - that is, within the normal limits - OCT values were higher than those of HRT. That small rim areas and volumes tended to be minimized by OCT, and larger ones to be magnified, was apparent also in the scatter plots (Figure 2Ab, Ac). For both OCT-DISC modes, differences falling within the \pm 1.96 SD span ranged considerably - for example, rim area OCT-DISC aut $_{\text {: }}$ from -0.81 to $1.18 \mathrm{~mm}^{2}$ (Figure $2 \mathrm{Bb}$ ); rim volume OCTDISC $_{\text {aut }}$ : from -0.52 to $0.54 \mathrm{~mm}^{3}$ (Figure $2 \mathrm{Bc}$ ) - which is likely to be of clinical relevance in the practice. In summary, Bland-Altman analysis revealed a poor agreement of OCT and HRT morphometric measures despite highly significant Pearson correlations.

\section{ONH measures and clinical diagnosis}

Quantitative ONH measures acquired with HRT, OCT-DISC $_{\text {aut, }}$ and OCT-DISC man $_{\text {are given in }}$ Table 4.

From an ophthalmoscopic/morphologic point of view, ONH's can be generally grouped into discs that have unremarkable cups, wide neuroretinal rim, and do not look glaucomatous, and discs that have large and deep cups, and look suspicious for glaucoma. From a clinical point of view, eyes can be grouped into such that have and such that do not have glaucoma. Optic disc imaging systems are meant to serve as topographic and diagnostic tools, so we interpreted HRT and OCT parameters in each context.

\section{HRT disc measures}

HRT disc area: In this study, eyes with normal looking discs (controls and OHT) had on average smaller ONHs than eyes with large cups (glaucoma-like discs or glaucoma). HRT cup area did not differ between normal controls and OHT eyes, and between glaucomalike and glaucomatous discs. HRT rim area was significantly smaller in the glaucoma eyes (mean $0.94 \mathrm{~mm}^{2}, P<0.01$ ), compared with each of the other three, nonglaucoma groups, those means lying between 1.34 and $1.40 \mathrm{~mm}^{2}$. Remarkably, largely excavated discs without clinical manifestations of glaucoma (glaucoma-like discs) exhibited a mean rim area within the normal range, ${ }^{1,27,28}$ and were thus not different from the rim area in the control group $(P=0.95)$. For HRT rim volume, a similar trend was observed: although decreasing from normal through the glaucoma-like discs, rim volume remained within the normal range in the three nonglaucoma groups, but was outside normal limits ${ }^{1,27,28}$ in the glaucoma group. In summary, HRT morphometric values characterized appropriately the categories of 'normally looking' and 'glaucomatous looking', and 'having glaucoma' vs 'not having glaucoma'.

\section{OCT disc measures}

In two eyes with small optic discs and small and shallow cups (HRT disc area 1.32 and $1.62 \mathrm{~mm}^{2}$; HRT cup-to-disc area ratio 0.02 and 0.04 , respectively), an erroneous rim area of $0.0 \mathrm{~mm}^{2}$ was calculated by OCT software in both automatic and manual mode (cup-to-disc area ratio of 1.0). For analysis, these values were replaced with rim areas equal to the disc areas, and cup-to-disc area ratio was corrected to 0.0 .

The systematic error found in the Bland-Altman analysis was reflected by consistently larger OCT-DISC aut $_{\text {at }}$ disc areas than those of HRT, independent of the clinical diagnosis. In accordance with the proportional error 
OCT-DISC and HRT

Scatter Plot $(n=49)$

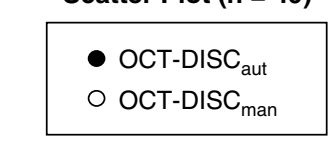

A

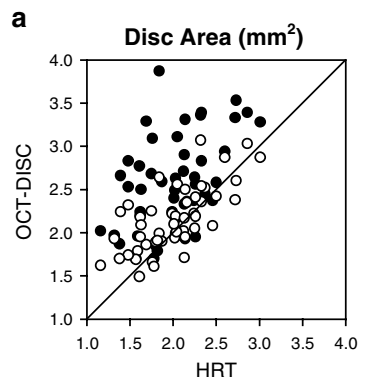

HRT

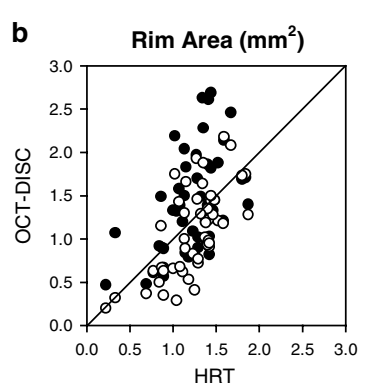

HRT

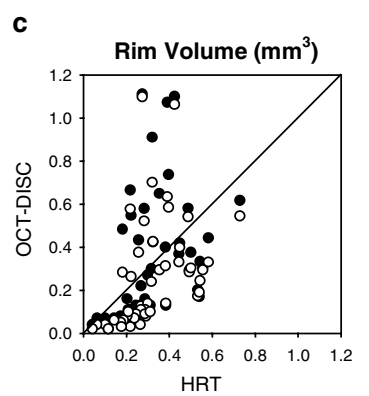

OCT-DISC $_{\text {aut }}$ and HRT

Bland-Altman Plot $(n=49)$

B
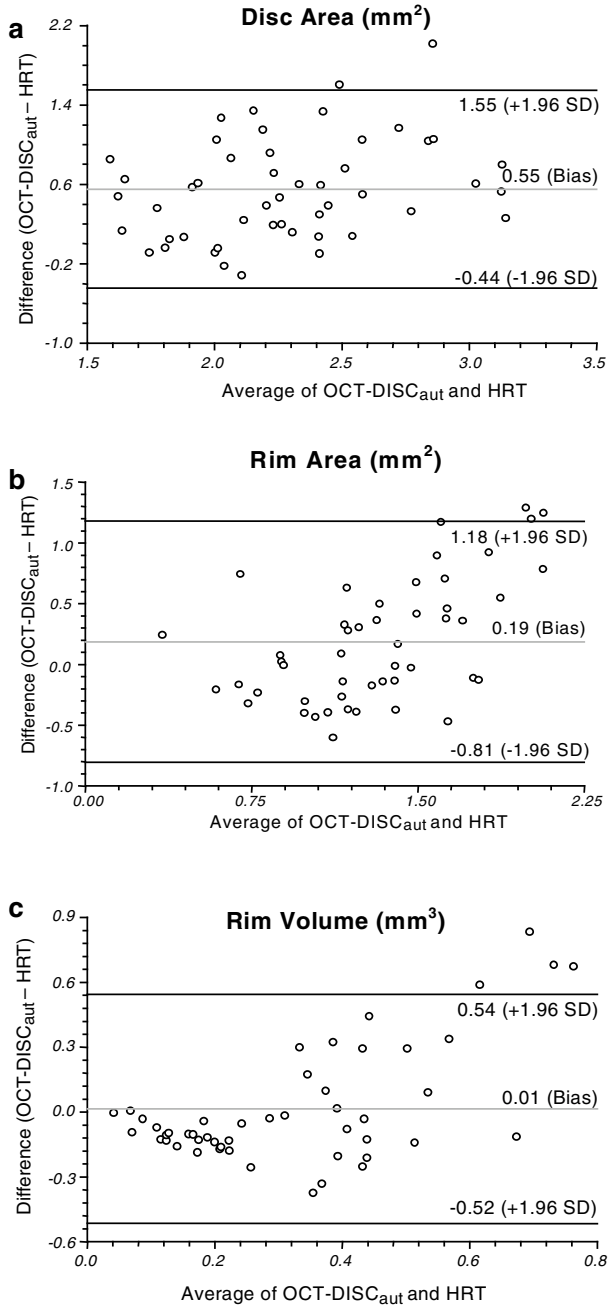

OCT-DISC $_{\text {man }}$ and HRT

Bland-Altman Plot $(n=49)$
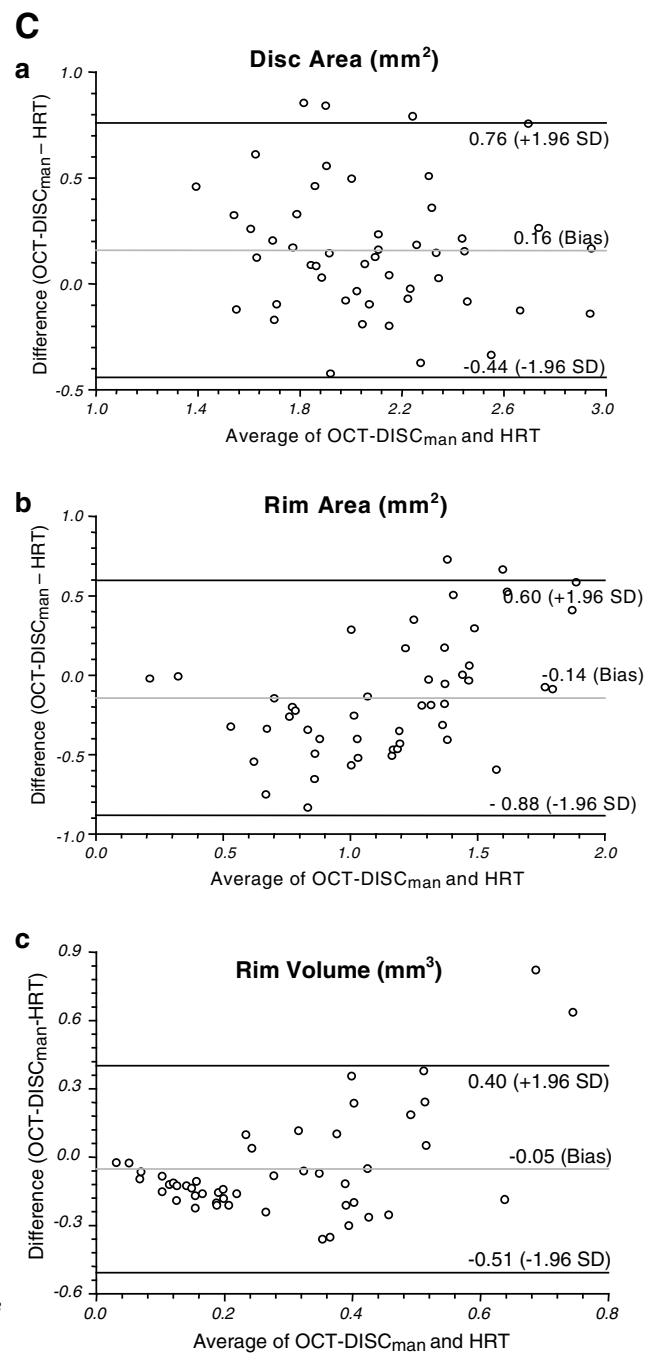

Figure 2 Scatter plots (A) and Bland-Altman plots (B, C) for comparison of disc area, rim area, and rim volume measures obtained by HRT (Heidelberg Retina Tomograph II) and OCT (Stratus OCT 3000) automated and manual disc mode (OCT-DISC aut $_{\text {and OCT- }}$ DISC $_{\text {man }}$ respectively). (A) The solid diagonal line represents the line of equality $(y=x)$. $(\mathrm{B}, \mathrm{C})$ The values for the mean, the mean $+1.96 \mathrm{SD}$ and the mean $-1.96 \mathrm{SD}$ of measure difference between both methods are included in the right part of each plot (bias, +1.96 $\mathrm{SD}$, and $-1.96 \mathrm{SD}$, respectively).

for rim area and rim volume reported above, OCT-DISC, especially in the automated mode, 'produced' magnified values in normal eyes and ocular hypertensives, and, compared with HRT, reduced values in glaucoma-like and glaucoma discs. As a consequence of this disbalance, extremely low OCT rim volumes in glaucoma were observed. Unlike with HRT, there was a significant difference in rim area and volume between the glaucoma-like discs and the other two, nonglaucoma groups $(P<0.015$ for all comparisons).

\section{Image-based classification of $\mathrm{ONH}$}

Table 5 describes the individual results and intra-eye combinations of the image-based classification of $\mathrm{ONH}$ as normal, borderline, and abnormal. It renders an 
Table 4 Clinical diagnosis and ONH measures obtained with HRT and OCT

\begin{tabular}{|c|c|c|c|c|}
\hline & $\begin{array}{l}\text { Normal } \\
(\mathrm{n}=14)\end{array}$ & $\begin{array}{l}\text { Ocular hypertensives } \\
\qquad(\mathrm{n}=9)\end{array}$ & $\begin{array}{l}\text { Glaucoma-like discs } \\
(\mathrm{n}=9)\end{array}$ & $\begin{array}{c}\text { Glaucoma } \\
(\mathrm{n}=17)\end{array}$ \\
\hline & Mean (SD) & & & \\
\hline \multicolumn{5}{|l|}{ Disc area $\left(\mathrm{mm}^{2}\right)$} \\
\hline HRT & $1.75(0.32)$ & $1.68(0.35)$ & $2.49(0.33)$ & $2.13(0.22)$ \\
\hline OCT-DISC $_{\text {aut }}$ & $2.29(0.50)$ & $2.50(0.29)$ & $2.86(0.46)$ & $2.66(0.65)$ \\
\hline OCT-DISC $_{\text {man }}$ & $1.89(0.28)$ & $2.09(0.26)$ & $2.51(0.34)$ & $2.24(0.35)$ \\
\hline \multicolumn{5}{|l|}{ Cup area $\left(\mathrm{mm}^{2}\right)$} \\
\hline HRT & $0.35(0.16)$ & $0.32(0.18)$ & $1.15(0.38)$ & $1.19(0.44)$ \\
\hline OCT-DISC $_{\text {aut }}$ & $0.51(0.27)$ & $0.52(0.35)$ & $1.65(0.68)$ & $1.75(0.63)$ \\
\hline OCT-DISC $_{\operatorname{man}}$ & $0.48(0.28)$ & $0.47(0.35)$ & $1.58(0.58)$ & $1.64(0.48)$ \\
\hline \multicolumn{5}{|c|}{ Cup-to-disc area ratio } \\
\hline HRT & $0.20(0.08)$ & $0.19(0.09)$ & $0.45(0.09)$ & $0.55(0.16)$ \\
\hline OCT-DISC $_{\mathrm{aut}}$ & $0.23(0.12)$ & $0.21(0.13)$ & $0.57(0.16)$ & $0.65(0.12)$ \\
\hline OCT-DISC $_{\text {man }}$ & $0.25(0.13)$ & $0.23(0.16)$ & $0.62(0.17)$ & $0.72(0.14)$ \\
\hline \multicolumn{5}{|l|}{ Rim area $\left(\mathrm{mm}^{2}\right)$} \\
\hline HRT & $1.40(0.25)$ & $1.36(0.30)$ & $1.34(0.08)$ & $0.94(0.34)$ \\
\hline OCT-DISC $_{\text {aut }}$ & $1.78(0.54)$ & $1.98(0.34)$ & $1.20(0.32)$ & $0.91(0.30)$ \\
\hline OCT-DISC $_{\operatorname{man}}$ & $1.41(0.27)$ & $1.62(0.39)$ & $0.93(0.32)$ & $0.60(0.25)$ \\
\hline \multicolumn{5}{|l|}{ Rim volume $\left(\mathrm{mm}^{3}\right)$} \\
\hline HRT & $0.43(0.13)$ & $0.34(0.13)$ & $0.30(0.08)$ & $0.21(0.12)$ \\
\hline OCT-DISC $_{\text {aut }}$ & $0.51(0.28)$ & $0.63(0.27)$ & $0.19(0.10)$ & $0.09(0.04)$ \\
\hline OCT-DISC $_{\text {man }}$ & $0.41(0.23)$ & $0.51(0.28)$ & $0.13(0.08)$ & $0.07(0.05)$ \\
\hline
\end{tabular}

For abbreviations, see Figure 1.

impression how a diagnosis was made with Stratus OCT according to the criteria suggested in Table 1, and how it corresponded with the diagnosis made with the 'standard' methods - disc photography and HRT. Statistically, good to very good agreement between all imaging methods was observed $(\kappa=0.66-0.89)$. Agreement was best between DISC-PHOT and HRT $(\kappa=0.89)$. OCT-DISC ${ }_{\text {man }}$ showed slightly better agreement with the photographic and HRT judge-

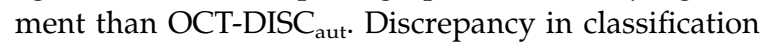
was more frequently observed with largely excavated discs (groups glaucoma-like and glaucoma), where OCT-DISC $_{\text {aut }}$ and OCT-DISC man $_{\text {tended to diagnose }}$ more 'aggressively', that is, borderline instead of normal, and abnormal instead of borderline.

\section{Discussion}

Our data show that the optic disc mode of Stratus OCT can reliably differentiate between normal and glaucoma, but its morphometric values cannot be directly compared with those of HRT. The problem, however, is that normative morphometric data are available, and have been established as clinically useful, from $\mathrm{ONH}$ morphometry of the past 25 years. ${ }^{19-23}$ It is therefore prudent for a new morphometric method to be assessed both for its ability to distinguish normality from abnormality, and to give interpretable and categorizable morphometric values.

\section{Automatic recognition of disc margin, disc size, and shape with Stratus OCT}

Once established in glaucoma care, OCT print-outs are likely to begin serving as image documents widely replacing other types of disc images, much the way HRT does now. It is therefore relevant that $\mathrm{ONH}$ size and shape are realistically depicted. In this study, we observed an erroneous automatic recognition of disc margin in $53 \%$ of the eyes, resulting in a pronounced disc shape and size misalignment. In a recently published work, Savini et $a l^{29}$ also reported an unsatisfactory automatic recognition of ONH edges in $61.1 \%$. The additional manual session gave us the opportunity to investigate this phenomenon more closely, and to analyse for associated factors. The role of PPA as a potential error source has been previously recognized. ${ }^{12}$ In our group, PPA turned out to be involved in less than $50 \%$ of the cases of failed disc margin recognition. Moreover, only $17 \%$ of manually corrected disc margin points were located within the PPA area. This clearly demonstrates that PPA is not the only, and maybe not the most 
Table 5 Classification of $\mathrm{ONH}$ as normal (N), borderline (B), or abnormal (A), and agreement between the imaging methods

\begin{tabular}{|c|c|c|c|c|c|}
\hline Clinical diagnosis & Eyes $(n)$ & DISC-PHOT & $H R T$ & OCT-DISC $C_{a u t}$ & OCT-DISC man $_{\text {m }}$ \\
\hline \multirow[t]{4}{*}{ Normal $(n=14)$} & 11 & $\mathrm{~N}$ & $\mathrm{~N}$ & $\mathrm{~N}$ & $\mathrm{~N}$ \\
\hline & 1 & $\mathrm{~N}$ & $\mathrm{~N}$ & $\mathrm{~N}$ & B \\
\hline & 1 & $\mathrm{~N}$ & B & $\mathrm{N}$ & $\mathrm{B}$ \\
\hline & 1 & $\mathrm{~N}$ & B & A & A \\
\hline \multirow[t]{2}{*}{ OHT $(n=9)$} & 7 & $\mathrm{~N}$ & $\mathrm{~N}$ & $\mathrm{~N}$ & $\mathrm{~N}$ \\
\hline & 2 & $\mathrm{~N}$ & $\mathrm{~N}$ & $\mathrm{~N}$ & $\mathrm{~B}$ \\
\hline \multirow[t]{7}{*}{ Glaucoma-like discs $(n=9)$} & 1 & $\mathrm{~B}$ & $\mathrm{~N}$ & $\mathrm{~N}$ & $\mathrm{~N}$ \\
\hline & 1 & A & B & $\mathrm{N}$ & B \\
\hline & 1 & $\mathrm{~B}$ & $\mathrm{~B}$ & $\mathrm{~B}$ & A \\
\hline & 1 & B & B & A & A \\
\hline & 1 & A & A & $\mathrm{B}$ & A \\
\hline & 1 & B & A & A & A \\
\hline & 3 & A & A & A & A \\
\hline \multirow[t]{4}{*}{ Glaucoma $(n=17)$} & 9 & A & A & A & A \\
\hline & 4 & A & A & $\mathrm{B}$ & A \\
\hline & 3 & B & B & A & A \\
\hline & $1^{\mathrm{a}}$ & $\mathrm{N}$ & $\mathrm{N}$ & $\mathrm{B}$ & $\mathrm{B}$ \\
\hline \multicolumn{6}{|l|}{ Agreement (kappa ${ }^{\mathrm{b}}$ ) } \\
\hline DISC-PHOT & & & 0.89 & 0.66 & 0.69 \\
\hline$H R T$ & & 0.89 & & 0.73 & 0.79 \\
\hline OCT-DISC $C_{a u t}$ & & 0.66 & 0.73 & & 0.77 \\
\hline OCT-DISC $C_{\text {man }}$ & & 0.69 & 0.79 & 0.77 & \\
\hline
\end{tabular}

For abbreviations, see Figure 1 and Table 1. Separated for clinical diagnosis, each line shows the number of eyes with an identical classification pattern. ${ }^{a}$ This subject had small discs that did not look glaucomatous on ophthalmoscopy; diagnosis of POAG was based on IOP 26-32 mmHg, central corneal thickness $520 \mu \mathrm{m}$, and reproducible visual field defects in the Bjerrum area; the partner eye is included in the line above.

${ }^{\mathrm{b} C}$ Cohen's kappa, weighted; $P<0.0001$ for all comparisons shown.

important, factor for suboptimal automated disc border recognition in OCT. Disc size and clinical diagnosis seemed not to play a role at all. Myopic refraction, on the other hand, tended to be more frequently associated with confusing automated placement of disc borders. Therefore, manual adjustment of disc margin points using a colour disc photograph as a reference is recommended with the present software, and may help obtaining a more realistic ONH image with the OCT.

\section{Correlation between disc measures in OCT automated and manual modes}

While manual fitting systematically reduced values for disc and rim area, it had little to no effect on cup area. This may explain to a certain extent the more 'aggressive'

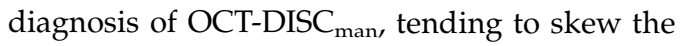
classification toward abnormal, particularly in eyes with big and deep cups. Since manual determination of RPE/choriocapillaris edges is doing what the software is supposed to do automatically, it should be assumed

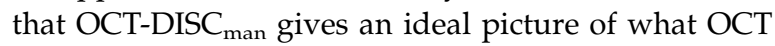
is designed to give. Evaluation with regard to morphometric and diagnostic reliability should therefore be based widely on OCT-DISC man $_{\text {results. }}$

In largely excavated discs (being the primary target of morphological evaluation in clinical practice), cups tend to be depicted too large, and rims too narrow. We speculate that reducing the cup-offset from $150 \mu \mathrm{m}$ to (roughly estimated) $50 \mu \mathrm{m}$ (ie, the cup margin would lie only $50 \mu \mathrm{m}$ above RPE) might improve OCT performance in these cases. Hrynchak et al also made the observation that if cup contour was positioned at $1 / 2$ of the total cup depth, that is, very close to the RPE level, OCT cup-to-disc ratio correlated much better with the stereoscopic clinical estimation than when cup contour was positioned at $1 / 3$ cup depth. ${ }^{30}$ On the other hand, a cup-offset of $50 \mu \mathrm{m}$ may lead to an unsatisfactory imaging of ONHs with very shallow cups, resulting more frequently in discs with no cupping. ${ }^{30}$ This seems to be an intrinsic characteristic of a method that uses as a reference level a structure (the $\mathrm{RPE}$, corresponding approximately to the outer edge of the scleral ring) that is relatively stationary in its $z$-axis, being independent of the retinal tissue thickness overlying it. 


\section{ONH measures and clinical diagnosis}

\section{HRT disc values}

In our group, HRT disc measurements correlated consistently with the clinical category and the clinical understanding - controls and ocular hypertensives exhibited normal values throughout; glaucoma eyes differed significantly and had abnormal parameters; glaucoma-like discs revealed cup areas comparable with the POAG eyes, but rim areas and volumes comparable with the normal eyes; glaucoma-like discs had the largest disc sizes.

\section{OCT disc values}

In the same context, there was a considerable difference in rim area and volume between normal and OHT eyes, on the one hand, and glaucoma suspects and glaucoma eyes, on the other (Table 4), in the transition of OCT parameters from normal to glaucoma. This difference was particularly striking regarding the rim volume, the

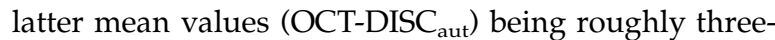
fold smaller in the glaucoma-suspect, and five to six-fold smaller in the glaucoma group compared to the other two groups. This stands in dissonance with what we would expect clinically. Baring in mind that the glaucomatous process is rather a continuum from normal to abnormal, the issue of cut-off values of these OCT parameters needs to be addressed. OCT rim volume in our study (both OCT-DISC aut $_{\text {and OCT-DISC }}$ man ) would have classified all glaucoma-like discs as clearly glaucomatous. The fact that manual placement of disc borders - that actually brought OCT disc shape and size much closer to the objectively observed in the $\mathrm{ONH}$ photographs - led to an increase of the above disproportion, may further implicate the necessity of adjusting the software algorithm with regard to cup-offset.

Based on our current understanding of anatomy, histology and morphometry, it is generally accepted that the scleral ring of Elschnig delineates the outer ONH borders. ${ }^{14-16,20}$ This rule was respected by us when determining HRT disc contour. Since the scleral ring is not imaged with the OCT, the algorithm is trained to locate disc border at the RPE/choriocapillaris edge. Histologically, in a 'typical' eye, RPE/choriocapillaris abuts on the outer edge of Elschnig's ring. Thus, by definition, optic discs imaged by OCT would appear larger compared to HRT. Theoretically, this difference should reflect the width and area of Elschnig's ring. There was, however, a much broader scatter of measurement differences, so that factors other than disc definition and anatomy should be sought to explain HRT/OCT discrepancies.

\section{Agreement between Stratus OCT and HRT II disc measures}

In their study in 2003, Schuman et al ${ }^{11}$ found a good correlation between HRT I and OCT 3 disc measures. We also observed statistically significant correlations between HRT II and Stratus OCT disc parameters, better

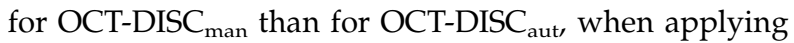
the Pearson correlation. However, most OCT-DISC mean values were systematically larger than those in HRT (Table 4). This held true for disc area, cup area and cupto-disc area ratio throughout the groups. OCT rim area and rim volume were larger than HRT values only in the eyes with normally looking optic discs (small and shallow cups, normal controls, and OHT), while in the eyes with glaucomatous looking discs (big and deep cups), OCT mean values were smaller than those in HRT. This 'change in direction' has not been reported previously. The Bland-Altman analysis revealed proportional errors and large dispersal of measurement differences between the two methods. Considering the latter as well as the above-discussed particularities in the definition of disc and cup margins, it does not seem likely that diagnostic ranges from planimetry and confocal scanning laser ophthalmoscopy could be adopted and continued in OCT disc imaging analysis.

\section{Differentiating between normal and abnormal optic discs}

Apart from the comparative analysis of topographic measures, we evaluated the 'practicability' of OCT disc diagnostic using masked assessment of the Stratus OCT print-out. In classifying optic nerve heads as normal, borderline, and abnormal, OCT-DISC protocol demonstrated an overall good agreement with the current standards for $\mathrm{ONH}$ evaluation, the colour photographs, and the HRT, although HRT II exhibited better consistency with the photographs. A limitation of this analysis is our definition of diagnostic criteria for normal and abnormal. Since there were no generally established discriminating criteria for OCT-DISC at the time of the study, and there was no integrated evaluation scale in its software, we adopted criteria based on HRT and ophthalmoscopy principles. We found thereby that when disc borders were satisfactorily depicted, macroscopic interpretation of the schematic disc and cup drawing in the OCT print-out enabled a diagnosis.

\section{Conclusions}

The OCT-DISC protocol performed overall well in differentiating between normal and glaucomatous ONHs, and showed, on the whole, a good diagnostic agreement with photographs and HRT. These positive 
diagnostic results stood in certain discrepancy with the frequent failure of disc border recognition, the latter accounting for unrealistic disc shapes and areas, and gross dissimilarities with HRT topographic values in some of the cases. A manual correction of disc borders was necessary more frequently, but not exclusively, in myopic eyes and eyes with peripapillary atrophy. According to the results of this study, morphometric parameters cannot be directly compared between HRT and OCT. OCT Disc mode tended to depict normal discs with wide neuroretinal rim as even 'more normal', and discs with a narrower rim and a large cup as even 'more abnormal', that is, it tended to enhance both normality and abnormality. Defining an own OCT-DISC normative database and possibly adjusting disc border recognition and cup-offset algorithms might further improve OCT morphometric performance.

\section{Acknowledgements}

We thank Carl Zeiss AG (Feldbach, Switzerland) for instructional and technical support during the study.

Financial interests: None.

\section{References}

1 Zangwill LM, Bowd C, Berry CC, Williams J, Blumenthal EZ, Sanchez-Galeana CA et al. Discriminating between normal and glaucomatous eyes using the Heidelberg Retina Tomograph, GDx Nerve Fiber Analyzer, and Optical Coherence Tomograph. Arch Ophthalmol 2001; 119: 985-993.

2 Wollstein G, Binnun E, Ben-Yosef N, Rozenman Y. Retinal thickness analyzer (RTA) of a imaging model eye and the optic nerve head - comparison with Heidelberg retina tomograph (HRT) measurements. In: Lemij H, Schuman JS (eds). The shape of glaucoma, quantitative neural imaging techniques. Kugler Publications: The Hague, 2000, pp 193-211.

3 Huang D, Swanson EA, Lin CP, Schuman JS, Stinson WG, Chang W et al. Optical coherence tomography. Science 1991; 254: 1178-1181.

4 Hee MR, Izatt JA, Swanson EA, Huang D, Schuman JS, Lin $\mathrm{CP}$ et al. Optical coherence tomography of the human retina. Arch Ophthalmol 1995; 113: 325-332.

5 Schuman JS, Hee MR, Arya AV, Pedut-Kloizman T, Puliafito CA, Fujimoto JG et al. Optical coherence tomography: a new tool for glaucoma diagnosis. Curr Opin Ophthalmol 1995; 6: 89-95.

6 Schuman JS, Hee MR, Puliafito CA, Wong C, PedutKloizman T, Lin CP et al. Quantification of nerve fiber layer thickness in normal and glaucomatous eyes using optical coherence tomography. Arch Ophthalmol 1995; 113: 586-596.

7 Blumenthal EZ, Williams JM, Weinreb RN, Girkin CA, Berry CC, Zangwill LM. Reproducibility of nerve fiber layer thickness measurements by use of optical coherence tomography. Ophthalmology 2000; 107: 2278-2282.

8 Kanamori A, Nakamura M, Escano MF, Seya R, Maeda H, Negi A. Evaluation of the glaucomatous damage on retinal nerve fiber layer thickness measured by optical coherence tomography. Am J Ophthalmol 2003; 135: 513-520.

9 Patella VM. Stratus OCT: Establishment of normative reference values for retinal nerve fiber layer thickness measurements. Carl Zess Meditec Inc.: Dublin, CA, 2003.

10 Paunescu LA, Schuman JS, Price LL, Stark PC, Beaton S, Ishikawa $\mathrm{H}$ et al. Reproducibility of nerve fiber thickness, macular thickness, and optic nerve head measurements using StratusOCT. Invest ophthalmol Vis Sci 2004; 45: 1716-1724.

11 Schuman JS, Wollstein G, Farra T, Hertzmark E, Aydin A, Fujimoto JG et al. Comparison of optic nerve head measurements obtained by optical coherence tomography and confocal scanning laser ophthalmoscopy. Am J Ophthalmol 2003; 135: 504-512.

12 Lai E, Wollstein G, Price LL, Paunescu LA, Stark PC, Fujimoto JG et al. Optical coherence tomography disc assessment in optic nerves with peripapillary atrophy. Ophthal Surg Lasers Imag 2003; 34: 498-504.

13 Iester M, Mikelberg FS, Courtright P, Drance SM. Correlation between the visual field indices and Heidelberg retina tomograph parameters. J Glaucoma 1997; 6: 78-82.

14 Vihanninjoki K, Tuulonen A, Burk RO, Airaksinen PJ. Comparison of optic disc measurements by Heidelberg Retina Tomograph and manual planimetric techniques. Acta Ophthalmol Scand 1997; 75: 512-515.

15 Jonas JB, Mardin CY, Grundler AE. Comparison of measurements of neuroretinal rim area between confocal laser scanning tomography and planimetry of photographs. Br J Ophthalmol 1998; 82: 362-366.

16 Garway-Heath DF, Poinoosawmy D, Wollstein G, Viswanathan A, Kamal D, Fontana L et al. Inter- and intraobserver variation in the analysis of optic disc images: comparison of the Heidelberg retina tomograph and computer assisted planimetry. Br J Ophthalmol 1999; 83: 664-669.

17 Miglior S, Casula M, Guareschi M, Marchetti I, Iester M, Orzalesi N. Clinical ability of Heidelberg retinal tomograph examination to detect glaucomatous visual field changes. Ophthalmology 2001; 108: 1621-1627.

18 Kiriyama N, Ando A, Fukui C, Nambu H, Nishikawa M, Terauchi $\mathrm{H}$ et al. A comparison of optic disc topographic parameters in patients with primary open angle glaucoma, normal tension glaucoma, and ocular hypertension. Graefes Arch Clin Exp Ophthalmol 2003; 241: 541-545.

19 Garway-Heath DF, Rudnicka AR, Lowe T, Foster PJ, Fitzke FW, Hitchings RA. Measurement of optic disc size: equivalence of methods to correct for ocular magnification. Br J Ophthalmol 1998; 82: 643-649.

20 Jonas JB, Gusek GC, Naumann GO. Optic disc, cup and neuroretinal rim size, configuration and correlations in normal eyes. Invest Ophthalmol Vis Sci 1988; 29: 1151-1158.

21 Libre PE. Association of disk size and glaucomatous cupping. Am J Ophthalmol 2003; 136: 398; author reply 398.

22 Budde WM, Jonas JB, Martus P, Grundler AE. Influence of optic disc size on neuroretinal rim shape in healthy eyes. J Glaucoma 2000; 9: 357-362.

23 Zangwill LM, Weinreb RN, Berry CC, Smith AR, Dirkes KA, Coleman AL et al. Racial differences in optic disc topography: baseline results from the confocal scanning laser ophthalmoscopy; ancillary study to the ocular hypertension treatment study. Arch Ophthalmol 2004; 122: 22-28. 
24 Bland M, Altman DG. Statistical methods for assessing the difference between two methods of measurement. Lancet 1986; i: $307-310$.

25 Fleiss JL, Nee CM, Landis JR. Large sample variance of kappa in the case of different sets of raters. Psychol Bull 1979; 86: 974-977.

26 Landis JR, Koch G. The measurement of observer agreement for categorical data. Biometrics 1977; 33: 159-174.

27 Iester M, Jonas JB, Mardin CY, Budde WM. Discriminant analysis models for early detection of glaucomatous optic disc changes. Br J Ophthalmol 2000; 84: 464-468.

28 Mardin CY, Horn F, Budde WM, Jonas JB. Morphometrische Querschnittsverlaufsbeobachtung der Papille mit dem HRT in Augen mit morphologischer Progression der glaukomatösen Atrophie der Papille des Nervus opticus. Klin Monatsbl Augenheilkd 2000; 217: 82-87.

29 Savini G, Zanini M, Carelli V, Sadun AA, Ross-Cisneros FN, Barboni P. Correlation between retinal nerve fibre layer thickness and optic nerve head size: an optical coherence tomography study. Br J Ophthalmol 2005; 89: 489-492.

30 Hrynchak P, Hutchings N, Jones D, Simpson T. A comparison of cup-to-disc ratio measurement in normal subjects using optical coherence tomography image analysis of the optic nerve head and stereo fundus biomicroscopy. Ophthalmic Physiol Opt 2004; 24: 543-550. 